\title{
Individual Differences in the Validity of a Cognitive Processing Model for Responses to Personality Inventories
}

\author{
Paul De Boeck \\ University of Leuven, Belgium
}

\begin{abstract}
An individual difference hypothesis was tested with respect to the validity of a vector type cognitive processing model for inventory responses. The validity index may be also considered an index of the conformity of inventory responses to the meaning structure of the items. Three short adjective and sentence type inventories were used, two of them consisting of mixed sets of items and one consisting of positive sets. It was concluded from the only low positive intercorrelations of the validities that individual differences are minor. Furthermore, evidence was found for an effect of the order of presentation of the inventories and for an effect of the inventory composition (positive or mixed), but not for a higher validity of the model for adjective than for sentence types of inventories.
\end{abstract}

Previous investigations concerning personality inventory responding have shown the utility of an intuitively plausible model according to which the respondent interprets the meaning of an item, compares it to what he/she knows about himself/herself, and expresses the comparison result in a response on a given judgment scale (Cliff, Bradley, \& Girard, 1973; Kuncel, 1973; Nowakowska, 1970; Rogers, 1974, 1978). The interpretation of an item and the selfknowledge both require cognitive representations that must be made in a common medium so as to allow for a comparison. Cliff et al. (1973)

APPLIED PSYCHOLOGICAL MEASUREMENT Vol. 5, No. 4, Fall 1981, pp. 481-492

(C) Copyright 1981 Applied Psychological Measurement Inc. $0146 \cdot 6216 / 81 / 040481 \cdot 12 \$ 1.60$ proposed a model for the common medium and the comparison processes. Item knowledge as well as the self-knowledge are supposed to be located in a common meaning space; and the response to an item is considered either a function of the scalar product of the item vector in the meaning space and the self-impression vector, i.e., the vector model, or it is considered a distance function of the item point and the self-impression point, i.e., the ideal point model (the self-impression point being the ideal point for an item to be endorsed).

The vector model has been shown to be fairly valid, and because of its simplicity and its only slight empirical inferiority to the ideal point model, it was considered as a good approximation of the true model (Cliff, 1977; De Boeck, 1978). The vector model formula of a response of subject $i$ to item $j$ reads as follows:

$x_{i j}=\sum_{m} w_{m i} v_{j m}+a_{i}$

in which $v_{j m}$ represents the coordinate of item $j$ on dimension $m, w_{m i}$ is the value of the self-impression of subject $i$ on dimension $m$, and $a_{i}$ is a constant to account for the overall level of endorsement.

The $v_{j m}$ values, i.e., the item coordinates, are derived independently of the inventory responses by a multidimensional scaling of the interitem meaning similarities. One group meaning space usually fits the data as well as a set of individualized spaces (Cliff, 1977; De Boeck, 1978). In 
accordance with the vector model formula, the meaning dimensions are to be considered linear predictors of the inventory responses of an individual so that they can be used in a linear regression analysis. The resulting regression weights are $w_{m i}$ estimators, and the multiple $R$ is an index of the model validity for the individual subject in question.

The validity of the vector model means that the items elicit responses in conformity with their meaning value on some basic dimensions. Moreover, the linear form of the vector model equation makes it equivalent to the component andilysis model variant of factor anaiysis, except for the additive constant of the vector model, so that it is not at all unusual to find the same meaning configuration dimensions among the item intercorrelation factors. The additive constant might add a general endorsement factor depending on the interindividual variance of the additive constant. As a matter of fact, the presence of the meaning dimensions among the correlational components also depends on the interindividual variance, namely, the variance of the dimensional self-impression values.

In general, however, the vector model leads to a reflection of the meaning dimensions in the componential or factorial structure of the inventory. That is, the degree of co-occurrence of inventory items in personality description is highly predictable from the meaning structure of those same items. For purposes of explication in the present paper, this phenomenon will be referred to as "meaning conformity." In fact, in studies where the vector model validity was fairly high, the meaning conformity phenomenon has been established (Cliff, 1977; Cliff et al., 1973; De Boeck, 1978). The vector model validity may also function as a measure of the extent to which individual subjects exhibit meaning conformity. The phenomenon of meaning conformity has aroused much interest in the recent literature (Block, Weiss, \& Thorne, 1979; D'Andrade, 1974; Gara \& Rosenberg, 1981; Shweder, 1977; Shweder \& D'Andrade, 1979), because of its bearing on the veridicality of perceived trait co- occurrences. Hence, an individual measure of meaning conformity, like the vector model validity, would be an interesting variable to be employed in research that is designed to reveal the determinants of the congruence in question.

For the present, three possible determinants of the vector model validity are examined in the literature: (1) instructions of faking or being candid, (2) the item type (adjectives or sentences), and (3) the inventory composition (positive or mixed, i.e., containing negatively as well as positively stated items). Faking instructions clearly heighten the individual meaning conformities (Cliff, 1977; Cliff et al., 1973), at least in mixed inventories. The evidence about the item type remains doubtful. Cliff (1977) has found lower meaning conformities using sentences instead of adjectives; but when adjective and sentence type inventories are made as equivalent as possible, and when they are administered at the same time in equivalent samples, the difference disappears (De Boeck, 1978). The mixed composition of the inventory (i.e., positively as well as negatively, or reversely, stated items) has yielded much higher meaning conformities than a positive composition (Cliff, 1977; i.e., without reversely stated items), probably because of a lower degree of differentation within subscales containing only positively stated items.

The present study examined whether there are individual differences with regard to meaning conformity. If so, this would imply that an individual difference (i.e., correlational) approach using variables such as cognitive style could be fruitful to this area and, hence, to the study of why there is a congruence between item meaning configurations and interitem correlations derived from personality judgments. It could be hypothesized, for example, that cognitively complex individuals or individuals who have high tolerance for ambiguity have lower degrees of meaning conformity than their counterparts. In fact, research along these lines has been started already (De Boeck, 1977) without an explicit test on the individual difference nature of meaning conformity. For the present purposes, three 
short personality inventories were used so that three multiple correlations were obtained for each individual.

\section{Method}

\section{Inventories}

The inventories that were used were taken from two previous studies. De Boeck (1978) has used a short version of the Dutch Five Personality Factors Test (5PFT; Elshout \& Akkerman, 1975), consisting of 20 items representing four of the five 5PFT subscales: Extraversion, Friendliness, Conscientiousness, and Neuroticism. Roughly half of the items from each factor were positively stated; the remaining items were negatively stated, yielding mixed item sets. The original inventory had sentence type items, but a parallel adjective type inventory was also developed. A list of the items of both types has been given by De Boeck (1978). The inventories appeared markedly equivalent with respect to the item meanings; and, as was expected, four meaning dimensions could be discerned in accordance with the factorial composition of the inventory. The two inventories were called PFT20am and PFT20sm, indicating the Personality $F$ actors $T$ est with $20 a$ djectives in $m$ ixed sets and the Personality Factors Test with 20 sentences in mixed sets, respectively.

On the basis of other SPFT items, a second short inventory was made up of four positively stated subscales, each subscale (which has four items) representing one of the four factors that are also represented in the PFT20am and the PFT20sm. A sentence type version was adapted to yield an adjective type equivalent. The resulting short inventories, PFT16ap (Personality $F$ actors $T$ est with 16 adjectives in positive sets) and PFT16sp (Personality Factors Test with 16 sentences in positive sets) are given in Table 1.

Finally, a third short inventory was composed from the Comrey Personality Scales (CPS), like the items used by Cliff (1977). The mixed set of Extraversion-Introversion (Shyness (Social Ease) ) items of Cliff was selected as a first sub- scale. A second subscale consisted of Cliff's mixed set of Empathy-Egocentrism (Empathy (Selflessness) ) items. Because the existing translation into Dutch is less appropriate, two items (the third Extraversion item and the first Empathy item) were substituted for original ones. In order to differentiate the short CPS version somewhat from the PFT20s and the PFT16s, a Masculinity-Femininity subscale was substituted for the Neuroticism subscale in the Cliff study (see Table 2). As for the other short inventories, an adjective type inventory, the CPS18am (Comrey Personality $S$ cales with 18 $a$ djectives in $m$ ixed sets), was construed to be an equivalent to the CPS18sm (Comrey Personality $S$ cales with 18 sentences in mixed sets). All of the selected CPS items and their adjectival equivalents are given in Table 2 .

In sum, six short inventories were used, three sentence type inventories and three corresponding adjective type inventories: the PFT20sm and PFT20am, which were both derived from the SPFT and contain four mixed subscales; the PFT16sp and PFT16ap, which were also derived from the 5PFT but contain four subscales with positively stated items; and the CPS18sm and CPS18am, which were both derived from the CPS and contain three mixed subscales.

For each short inventory, the instructions and response scales were borrowed from the original standard inventories from which they are derived.

\section{Meaning Similarity Ratings}

In order to obtain a measure of meaning structure as required in the vector model, meaning similarity judgments were gathered for the PFT16s and the CPS18s. The item meaning configurations for the PFT20s were taken from De Boeck (1978). For each of the PFT16s and the CPS18s, a booklet was arranged by presenting each of the items from the inventory at the head of a page above a list of items to be compared to the headed item with respect to their meaning, using a 9 -point scale $(1=$ very similar; $9=$ very 
Table 1

PFT16 Sentences and Adjective Type Items

Sentence Type

Adjective Type

Extraversion

1 Communicative. Entering easily into talk, to everybody.

2 Is always on the move, is involved in many activities.

3 Likes to be in the company of people; likes parties.

4 Likes to attract the attention of others; likes the spotlight.

Friendliness

1 Cooperative; ready to $f a l l$ in with a suggestion to cooperate, meeting complaints of others at least half way.

2 Considerate, kind. Thoughtful of others, full of friendly gestures.

3 Allows people what is desirable to them; free from jealousy.

4 Warm-hearted; makes an effort to act in everyone's best interest, whole-heartedly and with a smile.

Conscientiousness

1 Painstaking, precise; dislikes details being neglected; tends to be meticulous.

2 Conscientious, has strong principles; keeps to his words.

3 Tidy; never let his clothes Iie about. Everything has its proper place.

4 Persistent; presevering; as soon as he decides to do something, he spares neither pains nor time.

Neuroticism

1 Happens to be frequently and heavily excitable (infuriated, sad, in high spirits, love-sick, anxious, and so on).

excitable

2 Nervous; shows various signs of nervousness; restless movements, fumbling, being startled, palpatation of the heart, sleeplessness.

complaisant

friendly

benign

warm-hearted

3 Is in a state of severe tension when meeting

a problem; does not function optimally any

longer in such situations.

4 Weighed down, melancholic; feels frequently depressed

painstaking

conscientious

tidy

persistent

nervous

tense

depressed

dissimilar). The lists of items were composed in such a way that (1) each item had to be compared to each other, and that (2) a number of item pairs was repeated to check the individual reliability of similarity ratings (24 item pairs were repeated in a PFT16 booklet and 27 item pairs were repeated in a CPS18 booklet). The present method reduces to a short version of the 
Table 2

CPS18 Sentence* and Adjective Type Items

Adjective Type

Bxtraversion/Introversion

I (t) At a party I like to meet as many people as I can.

$2(+)$ It is easy for me to talk with people.

$3(+)$ I feel comfortable with people I have never even seen before.

4 (-) I try to avoid contacts with new people.

5 (-) I dislike being thrown together with people I don't know very well.

6 (-) I do less than share of talking in a conversation.

Empathy/Egocentrism

I (+) I enjoy helping people even if I don't know them very well.

$2(+)$ I am willing to share with others less for tunate.

3 (t) I prefer to look after the welfare of the ones I love before I worry about myself.

4 (-) I take care of myself before I think about other people's needs.

5 (-) I find it difficult to get interested in what is happening to other people.

6 (-) I would try to avoid a job in which I had to help people with their problems.

Masculinity/Femininity

I $(+)$ I could pick up a non-poisonous snake with my bare handa without being afraid.

2 (t) It would be hard to make me cry.

$3(+)$ A book about love and romance would bore me.

4 (-) A sad movie makes me feel like crying.

5 (-) I like movies which tell the story of two people in love.

6 (-) Seeing a lot of blood would make me feel faint.

outgoing

talkative

affable

seclusive

shy

silent

helpful

generous

unselfish

egoistic

unconcerned

uncooperative

fearless

imperturbable

sober

sensitive

romantic

feeble

*From Comrey, A.L. Comrey Personality Scales, Copyright 1970 by Educational and Industrial Testing Service, San Diego CA. All rights reserved, reproduced by permission.

conditional format with a meaning criterion that has been proposed by Girard and Cliff (1973).

\section{Subjects}

The subjects were 113 students in the final year of two high schools in the Dutch-speaking part of Belgium, 73 males and 40 females. They were randomly assigned to two groups, an
Adjective group of 57 students and a Sentence group of 56 students.

\section{Procedure}

The subjects were tested collectively in group sessions, and the anonymity of their responses was guaranteed. A first group, the Adjective group, completed the three short adjective type 
inventories and either the PFT16ap meaning similarity booklet $(N=29)$ or the CPS18am meaning similarity booklet $(N=28)$. The Sentence group completed the equivalent sentence type tasks with 27 and 29 subjects filling in the meaning similarity booklets of the PFT16sp and the CPS18sm, respectively. The self-judgment task was always given first, before the meaning similarity rating task, as this order is the least biasing one (see De Boeck, 1978).

The order of the self-judgment tasks, however, was varied so that four orders resulted: (1) PFT16-PFT20-CPS18, (2) PFT20-PFT16CPS18, (3) CPS18-PFI16-PFT20, and (4) CPS18-PFT20-PFT16. In the first two orders the SPFT-derived inventories preceded the CPS-derived inventory; in the remaining two orders the CPS-derived inventory came first. These are two levels of a first-order factor that is called PFT/CPS. In orders 1 and 3 the PFT16 preceded the PFT20, whereas in the orders 2 and 4 the PFT16 followed after the PFT20. This order factor is called PFT16/PFT20.

\section{Analysis}

There were four stages in the analysis: (1) a cluster analysis (Veldman, 1967) of individuals in order to identify possible meaning perception clusters; (2) nonmetric multidimensional scaling (MDS) analyses of meaning similarities (MINISSA; Roskam \& Lingoes, 1970); (3) multiple regression analyses with the degree of endorsement as a dependent variable and meaning dimensions (the outcomes of the MDSs) as the independent variables - the resulting multiple $R \mathrm{~s}$ are the indices of meaning conformity; and (4) an analysis of variance, using the BMDP2V program for an analysis including repeated measures (Dixon \& Brown, 1977) of the squared multiple correlations in accordance with $2^{5} \times 3$ design: five between-subject factors-sex, item type (adjectives or sentences), two order factors, and the meaning similarity judgment task (a PFT16 or CPS18 booklet)-and one within-subjects factor, the self-judgment inventory (PFT16, PFT20, or CPS18). The residual values derived from the analysis of variance were used to test the individual difference hypothesis, as those values were free from possible effects of the five between-subject factors (and their mutual interactions and interactions with the self-judgment inventory factor).

\section{Results}

\section{Cluster Analysis}

The cluster analysis was used to determine whether or not there were subgroups of subjects who differentially perceived the meaning structure of the various inventories. For these cluster analyses the similarity ratings for each subject were transformed into $z$ values using each subject's own mean rating. An analysis of the PFT16 half of the Adjective group $(N=29)$ revealed three clusters. The error index increased substantially from three to two clusters (from 170.2 to 243.1). Although the error was on the whole somewhat higher in the cluster analysis of the PFT16 half of the Sentence group $(N=26)$, a major increase in the error occurred at the transition from four to three clusters (from 172.2 to 196.7), so that four clusters were suitable. However, the average intracluster correlations were barely higher than the intercluster correlations for both groups. Moreover, a principle components analysis of interperson correlations yielded striking evidence for a one-factor solution in both cases, the respective eigenvalues being $12.28,1.43,1.29,1.22$, and 1.17 for the Adjective group; and $12.30,1.37,1.09$, and 0.97 for the Sentence group.

A similar result was obtained with regard to the CPS18s. The four clusters of CPS18am judges $(N=28)$ and the three clusters of CPS18sm judges $(N=27)$, which were chosen on the basis of error index increases from 142.2 to 158.6 and from 134.3 to 182.5 , respectively, did not identify clearly distinguished subject types, as the intracluster correlations approximated the values of the intercluster correlations. The homogeneity of each of the two meaning similarity judgment groups was confirmed by a prin- 
cipal components analysis yielding a very dominant first factor in each case, the respective eigenvalues being $8.27,1.53,1.43,1.38$, and 1.13 for the CPS18am subgroup; and 11.90, 1.36, $1.23,1.18$, and 1.02 for the CPS18sm subgroup. Viewed in the light of these results and those of previous research by Cliff et al. (1973), Cliff (1977), and De Boeck (1978), it seemed reasonable to do MDS based on similarities that were summed over the individual judges.

\section{Nonmetric MDS}

The stress values in the succeeding PFT16 scaling solutions containing five to one dimension(s) were $.025, .038, .072, .133$, and .281 for the adjective version and $.022, .039, .064, .113$, and .294 for the sentence version. Using the "elbow" criterion, a three-dimensional solution seemed the appropriate one in both cases, although four scales of the 5PFT were represented in the PFT16s. This should not be surprising, as the four item groups, if sufficiently homogen- eous, resemble the case of four points; and as for the representation of distances between four points, three dimensions always suffice. Moreover, these three dimensions produced the very neat differentiation of the four groups of items: Extraversion, Friendliness, Conscientiousness, and Neuroticism (see Table 3). The configurations of adjectives and sentences were fairly consistent with canonical correlations of $.929, .897$, and .675 (all $p$ 's $<.01$ ).

With respect to the CPS18s, three-dimensional solutions were also preferred on the basis of the following stress values from five to one dimension(s): $.047, .074, .106, .183, .392$ for the CPS18am and $.044, .062, .086, .130,0.312$ for the CPS18sm. After an additional orthogonal rotation, the three dimensions could be easily identified: Extraversion-Introversion, EmpathyEgocentrism, and Masculinity-Femininity (see Table 4). The scaling solutions of adjectives and sentences were fairly consistent with one another, as is shown in their canonical correlations: .942, .904, and .698 (all $p$ 's $<.01$ ).

Table 3

Meaning Configurations of PFT16 Adjective and Sentence Type Items

\begin{tabular}{|c|c|c|c|c|c|c|}
\hline & $\begin{array}{r}\text { Di } \\
\text { Adjed }\end{array}$ & $\begin{array}{l}\text { ension } \\
\text { ive Ty }\end{array}$ & $\begin{array}{l}\text { or } \\
\text { Items }\end{array}$ & $\begin{array}{r}\text { Di } \\
\text { Sent }\end{array}$ & $\begin{array}{l}\text { nsions } \\
\text { ce Type }\end{array}$ & tems \\
\hline & 1 & 2 & 3 & 1 & 2 & 3 \\
\hline Extraversion & & & & & & \\
\hline 1 communicative & -.557 & -.603 & -.390 & .560 & -.733 & -.183 \\
\hline 2 active & -.215 & -.210 & -.771 & .555 & -.111 & -.338 \\
\hline 3 convival & .358 & -.275 & -.692 & .631 & -.578 & -.367 \\
\hline 4 ostentatious & -.318 & -.902 & .048 & 1.182 & -.709 & -.004 \\
\hline Friendliness & & & & & & \\
\hline 1 complaisant & .648 & -.238 & -.455 & .330 & -.172 & -.734 \\
\hline 2 friendly & .449 & .256 & -.372 & -.106 & -.557 & -.585 \\
\hline 3 benign & .957 & -.210 & -.212 & -.666 & -.298 & -.648 \\
\hline 4 warm-hearted & .585 & -.400 & -.276 & -.094 & -.297 & -.615 \\
\hline Conscientiousness & & & & & & \\
\hline 1 painstaking & -.095 & .982 & -.283 & -.544 & .959 & .171 \\
\hline 2 conscientious & -.342 & 1.040 & .031 & -.542 & .573 & -.527 \\
\hline 3 tidy & .355 & .850 & -.227 & -.375 & .982 & .096 \\
\hline 4 persistent & -.509 & .596 & -.408 & -.041 & .933 & -.361 \\
\hline Neuroticism & & & & & & \\
\hline 1 excitable & .194 & -.571 & .730 & .093 & -.625 & .774 \\
\hline 2 nervous & -.746 & -.452 & .944 & -.025 & .017 & 1.105 \\
\hline 3 tense & -.811 & -.150 & .820 & -.222 & .223 & 1.078 \\
\hline 4 depressed & .049 & .286 & 1.514 & -.738 & -.235 & 1.138 \\
\hline
\end{tabular}


Table 4

Meaning Configurations of CPS18 Adjective and Sentence Type Items

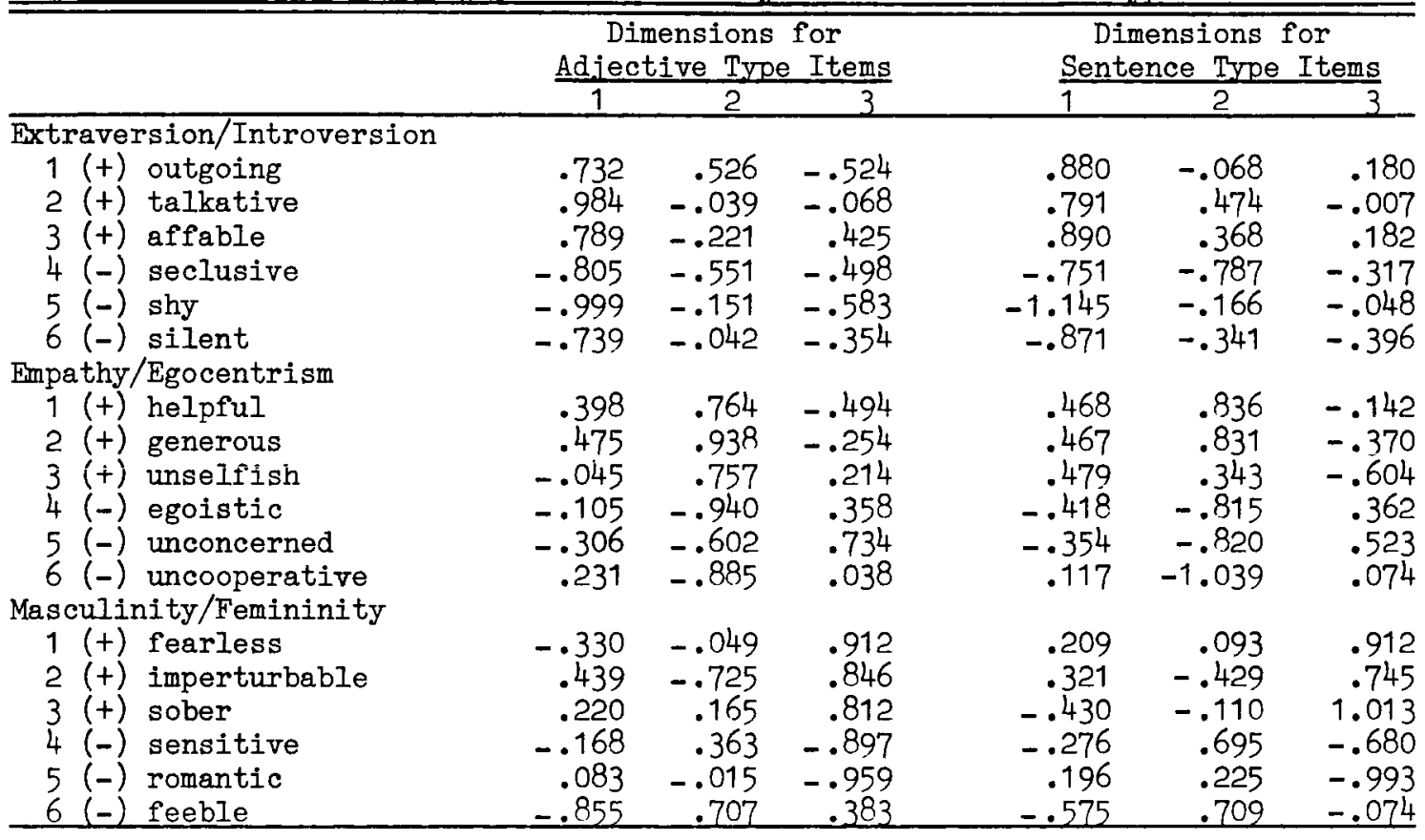

To present a possible distorting effect of unreliable similarity judgments, a MDS was done for each of the four short inventories after an elimination of ratings of unreliable judges from the similarity sums. A judge was considered unreliable if its individual reliability coefficient was not significant on the .05 level, one-tailed. However, irrespective of slightly lower stress values, essentially the same results were obtained. Mean reliabilities were $.618, .587, .509$, and .506 for the PFT16ap, PFT16sp, CPS18am, and CPS18sm, respectively.

\section{Regression Analyses}

Three regression analyses were carried out for each individual, with his/her endorsements as dependent variables and the meaning dimensions of the corresponding inventory as independent variables. With the PFT16s and the CPS18s, the dimensions of Tables 3 and 4 were used, respectively, and with the PFT20s the analyses were based on the dimensions from De Boeck (1978).

Median shrunken $R s$ were $.495, .639$, and .520 for the PFT16ap, the PFT20am, and the CPS18am, respectively, and $.474, .600$, and .493 for the corresponding sentence type inventories, respectively. The respective number of significant $R$ s $(p<.05)$ were 20,36 , and 26 out of 57 in the Adjective group and 22, 32, and 26 out of 56 in the Sentence group.

The $R$ level of the PFT20s is perfectly in conformity with the corresponding $R$ level in the De Boeck (1978) study, except for the slightly higher level of adjective $R \mathrm{~s}$ in comparison to sentence $R \mathrm{~s}$ in the present study. The level of CPS18 $R \mathrm{~s}$ should be comparable to the level of Cliff's $R s$ for candid responses to a mixed set of items, but it was much lower, whereas the $R \mathrm{~s}$ of the PFT16s exceeded those of the candid responses to positive set of items in the Cliff (1977) study. 


\section{Analysis of Variance}

An analysis of variance was done with the shrunken squared multiple correlations (i.e., the $R^{2} \mathrm{~s}$ corrected for number of predictors) as a dependent variable and with sex, item type, two order factors (the PFT/CPS and the PFT16/ PFT20 order factors) and the meaning similarity judgment task (a PFT16 or a CPS18 version) as between-subject factors, and the self-judgment inventory (PFT16, PFT20, and CPS18) as a within-subject factor. Because the matching of the inventories to the similarity judgment task was realized for only 108 subjects, the analysis of variance was based on 108 cases.

Among the between-subject factors, only sex had a significant effect $(F(1,76)=19.86$, $p<.01$ ), with a mean of .413 for females and .269 for males. The other significant effects concern the self-judgment task or inventory factor and its interactions with between-subject factors. Mean corrected $R^{2} \mathrm{~s}$ differed from inventory to inventory $(F(2,152)=13.04, p<.01)$, the means being .288, .411, and .267 for the PFT16, PFT20, and CPS18, respectively. Furthermore, there were significant interactions: Inventory $x$ PFT/CPS Order $\times$ PFT16/PFT20 Order $(F(2,152)=4.38, p<.05)$, Inventory $\times$ PFT16/PFT20 Order $\times$ Sex $(F(2,152)=5.03$, $p<.01)$, Inventory $\times$ PFT/CPS Order $\times$ PFT16/PFT20 Order $x$ Item Type $(F(2,152)=3.39, p<.05)$, and Inventory $x$ PFT/CPS Order $\times$ Item Type $\times$ Meaning Similarity Judgment Task $\times \operatorname{Sex} F(2,152)=3.49$, $p<.05)$.

Obviously, the order of presentation had an effect; an order factor was involved in each significant interaction. In order to have an idea of the order effects, the corrected $R^{2} s$ were transformed into deviations from their corresponding inventory mean, and then ordinal position means were derived for adjective as well as for sentence type inventories. The relation of the ordinal position of the inventory to the model validities appeared to be of an inverted U-shape, at least for adjective type inventories (see Figure
1). The strong curvature for adjectives is reflected in the four-way interaction: Inventory $x$ PFT/CPS Order $\times$ PFT16/PFT20 Order $\times$ Item Type, and even in the more general three-way interaction Inventory $x$ PFT/CPS Order $\times$ PFT16/PFT20 Order. These interactions may be understood by looking at the strongly rising lines in Figure 1 from position 1 to 2 if the adjective PFTs precede the adjective CPS, and at the strongly declining lines from position 2 to 3 if the adjective CPS version precedes the PFTs. The value of the PFT16 and PFT20 $R^{2}$ s closely depends on which one of the two precedes the other (PFT16/PFT20 order) and on whether the CPS18 comes first or not (PFT/CPS order). This applies primarily to adjective type inventories (item type factor).

It is shown in the interaction of inventory, PFT16/PFT20 order, and sex that the order effect on the difference between PFT16 and PFT20 also depends on the sex of the subjects. Indeed, when the PFT16 preceded the PFT20, the corrected $R^{2} \mathrm{~s}$ of the latter rose from .267 to .419 for males, and they dropped from .546 to .490 for females; conversely, when the PFT20 preceded the PFT16, the corrected $R^{2} \mathrm{~s}$ of the latter rose from .244 to .270 for males, whereas they dropped from .397 to .292 for females. The interaction of the highest order that is significant is left uninterpreted.

\section{Individual Differences}

As described above, a measure of meaning conformity (i.e., a multiple $R$ ) was obtained for each subject on each inventory (PFT16, PFT20, CPS18). To determine whether there are consistent individual differences in meaning conformity, the multiple $R \mathrm{~s}$ for each inventory were correlated across subjects (Spearman rank order correlations, $N=113$ ). Two of the correlations were significant: $.169(p<.05$, one-tailed) for the PFT16 and CPS18, and .316 $(p<.01$, onetailed) for the CPS18 and PFT20. The third intercorrelation, .051, for the PFT16 and PFT20 was not significant. 


\section{Figure 1}

Deviations from Inventory Means for Meaning Conformity

by Order Position of the Inventory, for Each Combination of Order Factors,

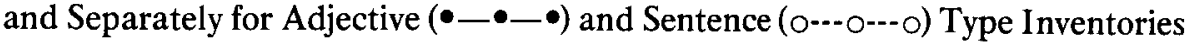

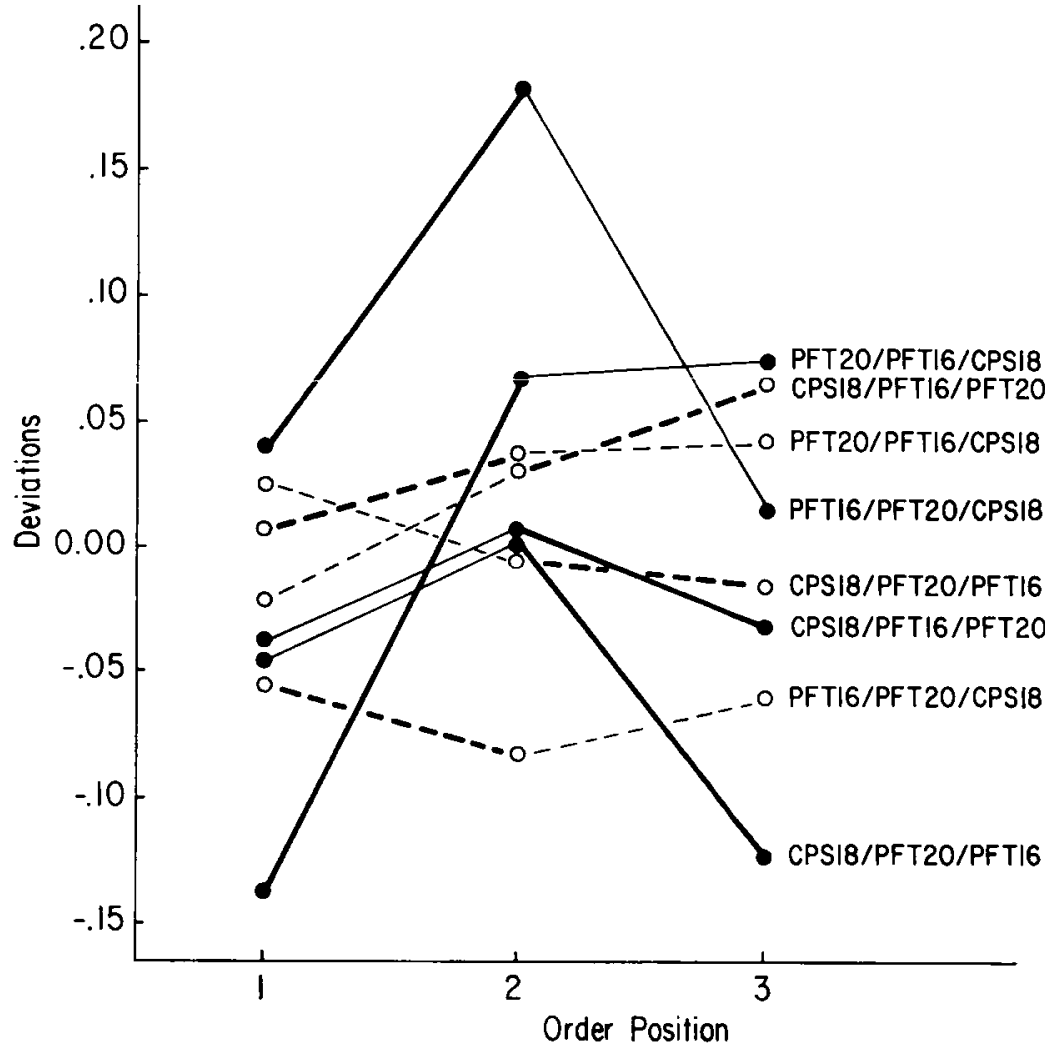

When, instead of uncorrected $R s$, the residuals of corrected $R^{2} \mathrm{~s}$ from the analysis of variance were used to test the individual difference hypothesis without confounding with possible group and treatment effects, essentially the same findings were arrived at, with Pearson correlations $(N=108)$ of $.162(p<.05$, one-tailed), $.235(p<.01$, one-tailed), and $.075(p<0.10)$ for the intercorrelations of the meaning conformity in the PFT16 and CPS18, the CPS18 and PFT20, and the PFT20 and PFT16, respectively.

When the same correlations were calculated for the Adjective group and the Sentence group, separately, a curious finding arises. The intercorrelations of both groups clearly diverged, the highest positive correlations in the one group be- coming the lowest (even negative) correlations in the other group (see Table 5). Also, the rank order correlations of the corresponding $R \mathrm{~s}$ show that pattern clearly.

\section{Discussion}

With respect to the individual difference hypothesis, no clear conclusions may be derived. The intercorrelations of individual meaning conformities were positive but not very high. When intercorrelations for the Adjective and Sentence groups were analyzed separately, the intercorrelations varied from -.177 to .509 (see Table 5).

The intercorrelations of the Sentence group are understandable from the number of charac- 
Table 5

Intercorrelations of Meaning Conformity Measures ${ }^{a}$

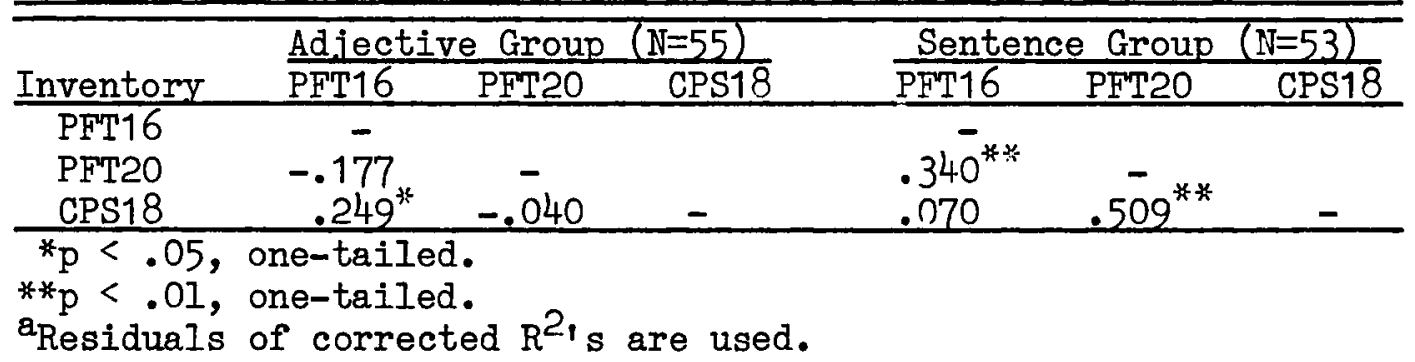

teristics the inventories have in common. There are two characteristics in which the three inventories can be similar or different, irrespective of the adjective or sentence type of the items. First, two of them contain mixed item sets, whereas the third consists of positive sets. Second, the PFT20 and the PFT16 are derived from the 5PFT, whereas the CPS18 is derived from the CPS. In summary, the PFT16 and the CPS18 differ on both of the characteristics, whereas the PFT16 and PFT20 as well as the PFT20 and CPS18 have one characteristic in common. In that way the near zero intercorrelations of the PFT16 and the CPS18 meaning conformities, and the fairly high other intercorrelations, might be accounted for. However, this interpretation implies that no general and stable individual differences exist, since when the inventories are enough differentiated, the intercorrelation of their meaning conformities disappears.

On the other hand, the correlations in the Adjective group might result from the order effects that have been established in the analysis of variance (see the interactions of inventory, sex, and item type with the PFT/CPS order and/or the PFT16/PFT20 order) working also on an individual level. These order effects may be considered an alternation phenomenon, i.e., low levels of meaning conformity rise and high levels fall in a subsequent inventory.

It might be hypothesized that (1) the alternation phenomenon is more pronounced the more similar the inventories are to each other, and that (2) the phenomenon is typical for adjective type inventories, as might be concluded from the analysis of variance and as might be accounted for by their less fixed denotative meaning. According to this hypothesis, the correlations between meaning conformities of adjective type inventories should be biased in a negative direction as a function of the similarity of the corresponding inventories. Hence, the lowest correlations must be expected where the highest positive correlations are found for sentence type inventories (at least under the expressed assumption that the latter correlations are a positive function of inventory similarity). This reversal of the correlation pattern is what in fact has been established.

From these findings that the meaning conformity phenomenon is so sensitive to order and that its degree is specific to the inventory in which it is measured, some hypotheses about its origin might be eliminated. First, meaning conformity is not a veridical reflection of a true consistency that pervades all domains of the behavioral reality of an individual subject. Second, the meaning conformity is not a result of an inevitable cognitive bias that is always operative. Moreover, whatever its origin is, the meaning conformity phenomenon could not be shown to be a clear individual difference variable in this study.

Furthermore, this study, designed to test an individual difference hypothesis, lends additional support to prior findings about possible meaning conformity differences connected with the inventory form. First, the item type did not 
have a significant effect. As in the study of De Boeck (1978), the validity of the vector model was not higher for adjectives than for sentences. Second, an inventory consisting of positive sets of items had a lower degree of vector model validity than an inventory consisting of mixed sets of items. Although the PFT16 and PFT20 are not as equivalent as possible, the lowering of the model validity in positive sets may be taken for granted, as it was found earlier and in a more pronounced way by Cliff (1977) under a more fully realized inventory equivalence.

To conclude, the evidence for individual differences with respect to the vector modei validity and therefore to meaning conformity is meager, except perhaps for sex differences, so that it seems more promising to concentrate further efforts on a study of situational determinants of meaning conformity.

\section{References}

Block, T., Weiss, D. S., \& Thorne, A. How relevant is a semantic similarity interpretation of personality ratings? Journal of Personality and Social Psychology, 1979, 37, 1055-1074.

Cliff, N. Further study of cognitive processing models for inventory response. Applied Psychological Measurement, 1977, 1, 41-49.

Cliff, N., Bradley, P., \& Girard, R. The investigation of cognitive models for inventory response. Multivariate Behavioral Research, 1973, 8, 407-425.

D'Andrade, R. G. Memory and the assessment of behavior. In T. Blalock (Ed.), Measurement in the social sciences. Chicago: Aldine-Atherton, 1974.

De Boeck, P. De invloed van cognitieve variabelen op de betekenisconformiteit van persoonlijkheidsbeoordelingen. (An investigation into cognitive factors in the meaning conformity of personality judgments, especially in personality inventory responding.) Unpublished doctoral thesis, University of Leuven, 1977.

De Boeck, $P$. Validity of a cognitive processing model for responses to adjective and sentence type inventories. Applied Psychological Measurement, 1978, 2, 369-376.

Dixon, W. J., \& Brown, M. B. (Eds.). BMDP-77. Biomedical Computer Programs. Berkeley: University of California Press, 1977.
Elshout, J. J., \& Akkerman, A. E. Vijf-Persoonlijkheidsfactoren Test (5PFT). Handleiding. (Five Personality Factors Test. Manual.) Nijmegen: Berkhout B. V., 1975.

Gara, M., \& Rosenberg, S. Linguistic factors in implicit personality theory. Journal of Personality and Social Psychology, in press.

Girard, R., \& Cliff, N. A comparison of methods for judging the similarity of personality inventory items. Multivariate Behavioral Research, 1973, 8, 71-88.

Kuncel, R. B. Response process and relative location of subject and item. Educational and Psychological Measurement, 1973, 33, 545-564.

Nowakowska, M. A model of answering to a questionnaire item. Acta Psychologica, 1970, 34, 420-439.

Rogers, T. B. An analysis of the stages underlying the process of responding to personality items. Acta Psychologica, 1974, 38, 205-213.

Rogers, T. B. Experimental evidence for the similarity of personality and attitude item responding. Acta Psychologica, 1978, 42, 21-28.

Roskam, E., \& Lingoes, J. C. A Fortran IV program for the smallest space analysis of squared symmetric matrices. Behavioral Science, 1970, 15, 204.

Shweder, R. A. Illusory correlation and the MMPI controversy. Journal of Consulting and Clinical Psychology, 1977, 45, 917-924.

Shweder, R. A., \& D'Andrade, R. G. Accurate reflection or systematic distortion? A reply to Block, Weiss, and Thorne. Journal of Personality and Social Psychology, 1979, 37, 1075-1084.

Veldman, D. J. Fortran programming for the behavioral sciences. New York: Holt, Rinehart, \& Winston, 1967.

\section{Acknowledgments}

I thank William Claeys, Michael Gara, and Gilbert Van der Steene for helpful comments on earlier drafts.

\section{Author's Address}

Send requests for reprints or further information to Paul De Boeck, Department of Psychology, Tiensestraat 102, B-3000 Leuven, Belgium. 